\title{
ENZYMES ET APPAREIL GUSTATIF LINGUAL CHEZ LE LAPIN DOMESTIQUE
}

\author{
L. ARVY \\ Station de Recherches de Physiologie animale, \\ Centre national de Recherches zootechniques, Jouy en Josas.
}

\section{SOMMAIRE}

Les bourgeons du goût linguaux du Lapin n'hydrolysent pas l'acétate d' $\alpha$-naphtol ; par contre ils hydrolysent fortement l'adénosine triphosphate, l'adénosine 5-monophosphate, l'acétyl et la buty. rylcholine.

Les glandules salivaires linguales annexées aux papilles gustatives, hydrolysent fortement le glycérophosphate de sodium en milieu acide, l'adénosinetriphosphate et l'acétate d' $\alpha$-naphtol. Leurs acini sont entourés d'un réseau de fibres qui hydrolysent l'acétyl-choline. En milieu alcalin le réseau vasculaire de ces glandules hydrolyse très fortement le glycérophosphate de sodium.

Les bourgeons du goût du Lapin ont été découverts, il y a près d'un siècle par LOVEN et SchwalBE. Ils ont été l'objet de très nombreuses descriptions, depuis les plus anciennes, macroscopiques et parfois approximatives, jusqu'aux plus réce ntes électroniques et extrêmement précises (LORENZO, TrujIr, C-CENOZ). Cependant, il est à peine exagéré de dire que nous ignorons tout de la perception des diverses sensations gustatives chez cet animal ; nous ne savons pas, en particulier, si chaque type de sensation est lié à un type déterminé de papille sensorielle, ni même s'il existe plusieurs types de papilles, en dehors des classiques filiformes, fungiformes, caliciformes et foliées.

D'ailleurs, le manque de corrélation entre la structure et la fonction n'est peutêtre nulle part plus apparent qu'en ce qui concerne les cellules neuro-sensorielles. Pour quelques Mammifères tels 1'Homme (RICH'TER), le Macaque (BLUN, Ruch, WalKer et Ectors), le Chat (Pfaffman), le Cobaye (Beiditer, Fishman et HARdiman), le Rat (PFAFFMAN), nous savons qu'ils sont parfaitement aptes à distinguer certaines saveurs grâce à des récepteurs particuliers dont les physiologistes ont fixé les localisations ; mais, les morphologistes, attardés au maniement des techniques de détection du système nerveux mises au point au siècle dernier (imprégnations argentiques, bleu de méthylène...), n’ont pas précisé la forme, le nombre ou la répartition de ces récepteurs. En outre, l'origine de la voie gustative est constituée par des fibres nerveuses extrêmement fines, non myélinisées de sorte que les techniques du type de celle de Marchi ne permettent pas leur étude. 
CrozIEr a émis l'hypothèse que chaque cellule neurosensorielle posséderait une substance réceptive spécifique avec laquelle se combinerait la substance stimulante pour l'activer. S'il en est ainsi on peut concevoir l'intervention d'une hydrolyse des particules goûtées, et, dans ce cas, des enzymes doivent être impliqués, sans doute de diverses manières, dans la perception des saveurs, à l'origine de la voie gustative dont le cheminement a, par ailleurs, été élucidé (BREMER, Ectors, GEREBTzOFF).

Il semble donc que les techniques histoenzymologiques en apportant de nouvelles données morphologiques pourraient aider à l'élaboration de quelque hypothèse sur les mécanismes qui président à la gustation.

\section{MATÉRIEL E'T MÉTHODES}

Les détections enzymatiques ont été faites sur des coupes à la congélation, transversales et parasagittales, de langue de Lapins de divers âges, fixées par le formol à ro p. ioo neutre et froid, pendant peu de temps (toujours moins de 6 heures). Elles ont été traitées par les méthodes de Gomori pour les phosphomonoestérases alcaline et acide, par les méthodes de Wachstein et Meisel pour l'adénosine 5'-monophosphatase et l'adénosine-triphosphatase, par la méthode de Burstone pour l'acétylnaphtolestérase, par une variante de la technique de Koelle-Gerebtzoff pour l'acétylthiocholinesterase et la butyrylthiocholinestérase. La recherche des oxydases s'est limitée à la cytochromoxydase (réaction nadi) et à l'histaminoxydase, (technique personnelle (1); les deux recherches n'ont révélé qu'une activité très faible et même douteuse. L'activité scucinodéshydrogénasique trahit l'existence des plis de l'organe folié, par un fin liseré pourpre de formazan, lorsqu'on applique sur l'organe cntier le bain de Farber et Louvière.

\section{RÉSULTATS}

La détection des activités phosphomonoestérasiques alcaline et acide (méthodes de Gomori) ne fait pas apparaître les centres récepteurs sensoriels. L'activité enzymatique est localisée au niveau des papilles gustatives comme au niveau du reste de la muqueuse linguale c'est-à-dire sur les vaisseaux et sur la basale malpighienne pour l'activité alcaline et seulement sur la basale pour l'activité acide.

La recherche d'une activité acétylnaphtolestérasique reste également négative ; les bourgeons du gôtt apparaissent en clair dans le reste de l'épithélium riche en cet enzyme.

Les activités adénosine 5-monophosphatasique, adénosine triphosphatasique ou cholinesthérasique révèlent électivement les centres neuro-sensoriels linguaux ; leurs activités enzymatiques sont telles qu'ils tranchent sur le reste de l'épithélium lingual, pauvre en ces enzymes. Ils apparaissent avec des aspects très différents suivant leur localisation et suivant le substrat mis en œuvre.

On retrouve sur les coupes, les papilles gustatives visibles à l'examen macroscopique. Ce sont d'arrière en avant :

ro les deux papilles caliciformes situées de part et d'autre de la ligne médiane, au niveau de l'insertion des piliers du voile du palais,

$2^{\circ}$ les deux organes foliés situés de part et d'autre des précédentes et sur un plan plus antérieur ; ils sont ovoïdes, à pointe un peu plus effilée vers l'arrière que vers l'avant, à grand axe oblique de haut en bas et d'arrière en avant; ils tranchent sur le reste de la muqueuse linguale rosée par leur teinte ; ils sont en effet d'un blanc

(1) Les techniques actuelles d'histoenzynologie, Biol. méd. 1957, 46, 448. 
translucide et pourvus de I5 à I7 fines stries d'un blanc opaque, à peu près régulièrement espacées; quelques stries à point de départ antérieur n'atteignent pas le bord postérieur. Les stries sont perpendiculaires au grand axe de l'organe folié ; elles signalent les invaginations épithéliales qui délimitent les feuillets de l'organe,

$3^{0}$ quelques grosses papilles verruqueuses, en saillie sur la pointe de la langue, formées d'un paquet de bourgeons du goût,

$4^{0}$ de nombreuses papilles filiformes réparties sur toute la face supérieure de la langue, avec, parmi elles et très clairsemées, des papilles fungiformes.

L'histoenzymologie révèle des aspects-propres à chaque type de papille, liés aux particularités de sa vascularisation et de son innervation vasculaire.

\section{I - Papilles caliciformes et organes foliés:}

Les papilles caliciformes, les faces opposées des plis de l'organe folié, la surface des papilles fungiformes et toute la périphérie des grosses papilles verruqueuses portent de très nombreux bourgeons du goût. Ils se signalent par leur forte activité

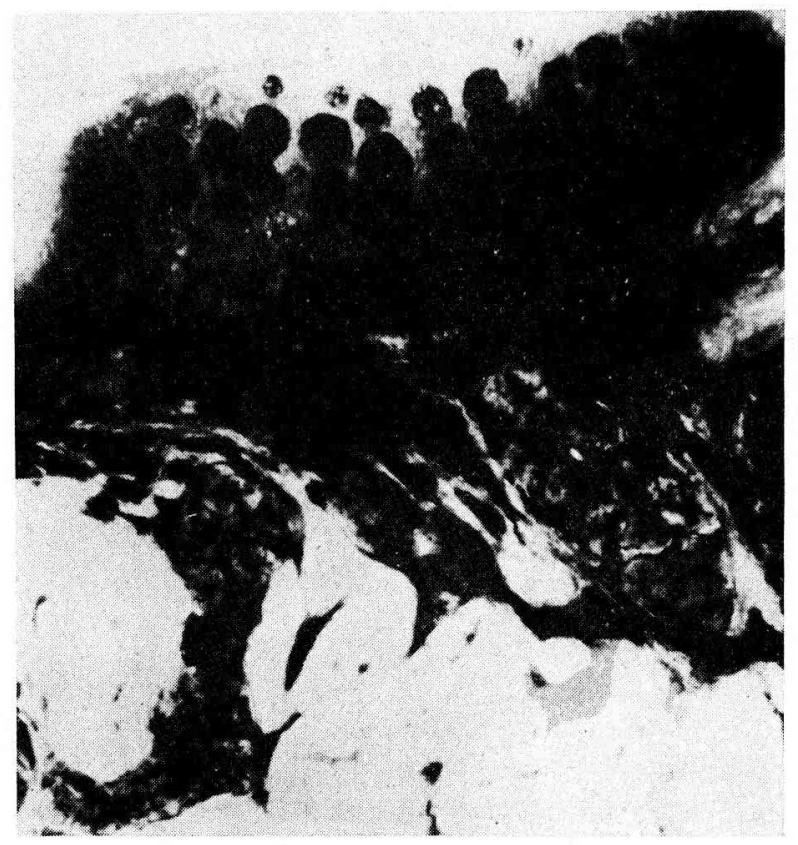

FIG. I - Coupe oblique d'une papille caliciforme après détection de l'activité adénosine-triphosphatasique, avec de nombreux bourgeons gustatifs recouvrant la face interne. A la base de la coupe, en noir, des artères, en gris :les acini des glandules salivaires annexées à la papille.

adénosine triphosphatasique et leurs fortes activités cholinestérasiques. Ces deux activités sont liées à l'existence au niveau des bourgeons d'un réseau vasculaire d'une finesse et d'une densité extrême ; ces vaisseaux comme les artères dont ils dérivent, ont une forte activité adénosine-triphosphatasique qui leur est propre ; ils supportent, en outre, un riche réseau cholinergique qui les entoure. 
Au niveau des papilles caliciformes les artères afférentes se ramifient à l'extrême ; l'ensemble de leurs ramifications s'ordonne en une sorte de treillis formant une coupe évasée, dont les faces interne et externe se dépriment en petites cupules qui enserrent les bases des bourgeons du goût (fig. I).
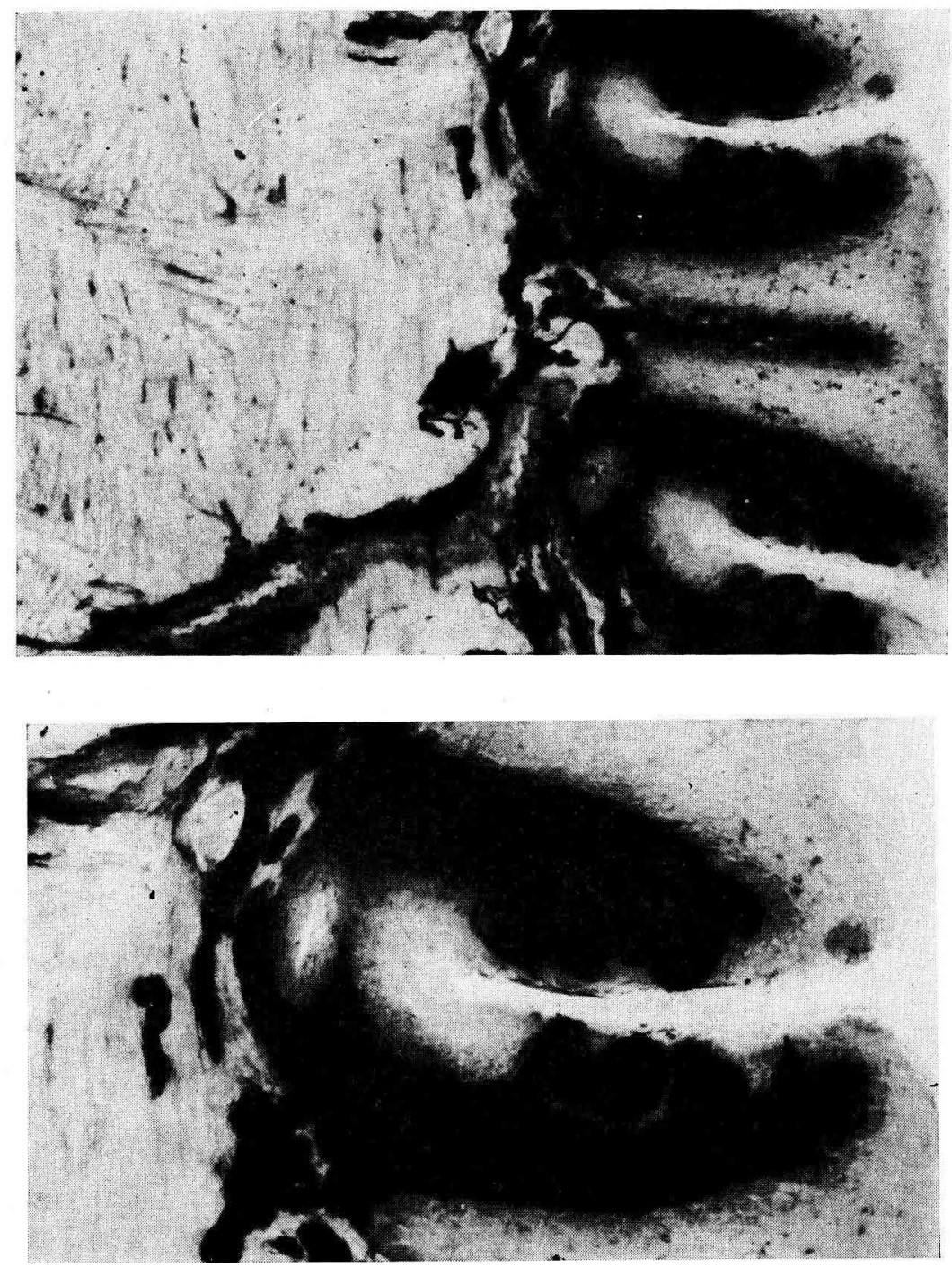

FIG. 2 - Coupe parallèle au grand axe de l'organe. - En haut : un feuillet entier avec ses deux plis épithéliaux, sur les parois desquelles s'ouvrent les pores des bourgeons gustatifs. Au fond des plis, en gris, la section des canaux excréteurs de glandules salivaires; en noir la répartition de l'activité adénosinetriphosphatasitque. - En bas : fort grossissement d'un des plis ( $\mathrm{pH} 7,2$, incubation 15 minutes).

Au niveau de l'organe folié les artères afférentes montent parallèlement aux plis épithéliaux de part et d'autre de l'axe dermique du feuillet (fig. 2 ) ; la paroi médiale de l'artère est régulière mais la paroi pré-gemmale donne un grand nombre de ra- 
meaux qui enserrent la base des bourgeons gustatifs en autant de cupules; un certain nombre de branches pénètrent dans les bourgeons ; elles s'y recourbent en anse ou s'y anastomosent en un véritable réseau admirable qui s'étend jusqu'au canal gustatif, à l'extrémité proximale duquel elles forment une sorte de collier. Le pore gustatif lui-même a une forte activité adénosine-triphosphatasique.

La recherche des activités acétyl ou butyrylthiocholinesthérase révèle des aspects analogues car tout le réseau artériolaire des papilles gustatives est riche en fibres cholinergiques.

Les axes dermiques des papilles caliciformes et des plis de l'organe folié se signalent par des vaisseaux veineux qui ont une forte activité adénosine, 5'-monophosphatasique.

Au fond des plis épithéliaux qui contiennent les bourgeons gustatifs débouchent les canaux excréteurs de glandules salivaires linguales riches en phosphomonoestérase acide et en adénosine-triphosphatase. Ces glandules ont quelque activité acétylnaphtolestérasique et cholinestérasique ; un riche réseau de fibres cholinergiques entoure leurs acini.

\section{2 - Papilles verruqueuses, fungiformes et fliformes:} lites.

Ces papilles ont la particularité d'être dépourvues de glandules salivaires satel-

Les premières portent des bourgeons du goût sur toute leur surface ; les papilles fungiformes n'ont de bourgeons du goût que sur leur face linguale et les papilles filiformes en sont dépourvues.

Ces trois sortes de papilles ont des activités enzymatiques différentes liées à leurs réseaux vasculaires différents. Les réseaux veineux sont riches en adénosine 5 -phosphatase; ils se déversent dans des veinules linguales sinueuses, également riches en cet enzyme; ils occupent l'axe dermique des papilles filiformes alors qu'ils se répartissent à la périphérie du derme dans les papilles fungiformes et verruqueuses. Les réseaux artériels, issus d'artérioles dorsolinguales, sont digités au niveau des papilles fungiformes, en pommes d'arrosoir au niveau des papilles verruqueuses et réduits à quelques anses au niveau des papilles filiformes ; mais dans tous les cas ces vaisseaux riches en adénosine-triphosphatase sont entourés d'un lacis de fibres cholinergiques, comme les bourgeons du goût qui les prolongent.

\section{DISCUSSION}

Les localisations enzymatiques, satisfaisantes pour l'esprit, que je viens de rapporter prouvent encore une fois, qu'il est rationnel d'utiliser en histoenzymologie des substrats peu concentrés, des incubations courtes, des $\mathrm{pH}$ voisins de la neutralité, en vue de faire travailler l'enzyme, éventuellement présent, dans des conditions voisines de celles où il travaille in vivo.

BOURNe (I9I8), BARAdi et BOURNE (I953) ont déjà signalé l'existence d'enzymes variés au niveau des bourgeons du goût ; ils ont cru déceler une adénosine-triphosphatase, une glucose-6-phosphatase, etc. sur des tissus fixés par l'acétone, coupés à la paraffine et mis en incubation avec les substrats adéquats, à pH 9. I1 est évident aujourd'hui 
qu'il est vain d'espérer retrouver quelques traces d'enzymes aussi labiles après un tel traitement. De même que l'orsqu'on observe une hydrolyse de l'adénosine 5'-phosphate, à $\mathrm{pH} 9$, il est peu vraisemblable qu'elle soit due à la $5^{\prime}$-nucléotidase puisque le pH optimal de cet enzyme est de 7,2-7,3 (ORUNESU, I944, I955).

Quel peut être le rôle d'une activité adénosinetriphosphatasique aussi considérable au niveau des bourgeons du goût? On peut admettre que l'hydrolyse de divers composés modifie l'équilibre ionique et par suite la perméabilité cellulaire, la vasoconstriction, ou la vaso-dilatation, génératrices de variations et de pression sur les extrémités nerveuses.

Reçu en avril 1959.

\section{SUMMARY}

ENZYMES AND THE LINGUAL TASTE BUDS IN THE TAME RABBIT.

The taste buds of the rabbit's tongue are inactive on $\alpha$-napthol acetate. On the other hand they are very active on adenosinetriphosphate, adenosine- 5 monophosphate, acetyl and butyrylcholine.

The lingual salivary glandulae joined to the taste buds hydrolyse glycerophosphate in acid media and also adenosine-triphosphate and $\alpha$-naphtol acetate. Their acini are enclosed in meshes of fibers which hydrolyse strongly acetylthiocholine. In alkaline media the wall of the blood vessels of these glandulae act strongly on sodium $\beta$ glycerophosphate.

\section{RÉFÉRENCES BIBLIOGRAPHIQUES}

ARnstein C., I893. Die Nervenendigungen in dem Schmacksorgan des Süuger. Arch. Mikr. Anat,. 41. I95.

ARVY L., 1959. Contribution à l'histoenzymologie des chémorécepteurs linguaux chez le Mouton domestique. C.R. Acad. Sci., 248, 306r.

Baradi A. F., Bourne G. H., I953. Gustatory and olfactory epithelia. Internat. Rev. Cytoloy, 2, $289-330$.

Beidler L. M., Fishman I. Y., Hardiman C. W., I955. Species differences in taste responses. Amer. J. Physiol., 181, 235-239.

BLum M., Ruch T. C., Walker A. B., I943. Localisation of taste in the thalamus of Macaca mulatia. Yale J. Biol. Med., 16, I75 191.

Botrne G. H., 1948. Alkaline phosphatase in taste buds and nasal mucosa. Nature, 161, 445.

Bremer F., 1923. Centre cortical du goût chez le Lapin. C. R. Soc. Biol., 89, 432.

CAjAt S. Ramon, igog. Histologie du système nerveux de l'Homme et des Vertébrés. Maloine édit., Paris, lome I, 725 .

Crozier W. J., 1934. Chemoreception, chap. 19 in : A Handbook of general experimental psychology. Murchisson Worchester édit. Clark Univ. Press Mass.

EcTors L.. I9.36. Étude de l'activité électrique du cortex cérélsral chez. le Lapin non narcotinisé ni curarisé. Arch. int. Physiol. 43, 267-208.

Fisari R., Panacci, i 89 . Les terminaisons des nerfs dans la muqueuse et dans les glandes séreuses de la langue. Arch. ital. de Biol., cité par CajAL.

GerebtzofF M. A., J939. Les voies centrales de la sensibilité et du goût et de leurs terminaisons thalamiques. Ia Cellule, 48 , 91-r 46.

Gerebtzofr M. A., т 94I. Recherches oscillographiques et anatomopathologiques sur les centres cortical et thalamique du goût. Arch. int. Physiol., 51, i99-2то.

Heideniain M., igi4. Uber die Sinnesfelder und die Geschmacksnospen der Papilla foliata des Kaninchens. Arch. mikr. Anat., 85, 365-479.

LaRseli. O.: I95r. Anatomy of the nervous system. Appellon édil., Nez-York.

LENHOSSEK J. (von). ז89.. Beiträge zur Histologie des Nerven systems und der Sinnesorgane. Wiesbaden.

Lorenzo A. T. (de) ig 58 . Eiectron microscopic observations on the taste buds on the Rubbit. $J$ Biophys. Biochim. Cylol 4. 14.3-148.

Lovés O. Ch., I867. Bildrag till Kännedomen om tungaus smakpapiller. Medicinskt Archiv, 3, r-14 et Anatomische und physiologische Arbeiten Herausgegeben ram R. TIGERSTEDT, Leipzig, I906 p.I53-68. ORunesu M., r953. pH ed attivita 5-nucleotidasica. Boll. Soc. ital. Biol. sper., 29, I 707.

Orunesu M., Fieschi C., I955. Ricerche sulla 5-nucleotidisi epatica. Boll. Soc. ital. Biol. sper., 31, $478-479$.

PATTON H. D., RuCi T. C., WALker A, E., I944. Experimental hypogenesia from Horsley-Clark lesions of the thalamus of Macaca mulatta. J. Neurophysiol., 7, I 7 Y-I84. 
Pfaffmann C., I94I. Gustatory afferent impulses. J. Cell. comp. Physiol., 17, 243-258.

Pfaffmann C., I953. Species differences in taste sensitivity. Science, 11\%, 470.

RichteR C. P., r943. Total self regulatory functions in animals and human beings. Harvey Lecture, 38, $63-103$.

Retzius G., I892. Die Nervenendigungen in dem Geschmacksorgan der Saugethiere und Amphibien. Biol. Untersuchungen, 4.

Schwalbe G., I867. Das Epithel des Papillae vallatae. Arch. mikr. Anat., 3,, 504-508.

TRUjlllo-CrNoz O., i957. Electron microscopy of the rabbit gustatory buds. Z. Zellf., 46, 272-280. 